personal experience, which affects his wife and then his brother. As we all might when faced with a cancer diagnosis, he mulls over their personal risk factors and the evidence for these. He unravels their sobering journey with an unsentimental but touching frankness.

The epilogue explains the jacket sleeve; the words of the title intertwine with the bindweed Convolvulus arvensis. Cancer is a tenacious weed; carefully dissected and subject to chemical attack, only to return and seed stealthily at the next opportunity. For the present moment at least, chaos remains the natural state of matter - any triumph over cancer is temporary.

\section{Jenni Gibson,}

GP Partner, Wishaw Health Centre, Wishaw.

E-mail: jennigibsonahotmail.co.uk

DOI: 10.3399/bjgp14X677617

$$
* * * * *
$$

\section{TRUE DOCTORING}

\section{Being a Doctor:}

\section{Understanding Medical Practice}

Hamish Wilson and Wayne Cunningham Otago University Press, 2013

PB, 276pp, E12.50, 978-1877578366

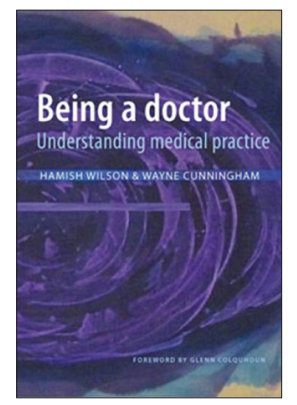

In Being a Doctor Hamish Wilson and Wayne Cunningham explore what doctoring is, bringing in elements often misunderstood or undervalued in medical practice, even though they are of vital importance.

They start by discussing the concepts of wellness, illness, and disease; suffering, healing, and curing; and how the doctor's role, where cure is often not possible, is one of healing through facilitating the patient's movement towards wholeness and personal integrity'. Here is the need for a social constructivist as well as a biomedical approach: emotional as well as biomedical intelligence. Two particular examples bring these approaches to light. The first is the 'heartsink experience' where the awareness of transference and countertransference can assist in the understanding of what is going on for both the patient and the doctor. The second is how to approach illness where there is seemingly no disease, true somatisation, or where we have not yet been able to make a diagnosis.

The next part of the book addresses the culture of medicine, not always healthy; and adverse outcomes and patient safety with the need to put focus on systems rather than just individuals. In navigating all this the authors underline the importance of reflective practice, and the need for looking after ourselves as doctors. Finally comes a chapter on the place of the doctor in the future. There are challenges ahead and we need to involve ourselves in preparing to meet them. The key to a good healthcare system, reiterated throughout the book, is the coaching and mentoring of our undergraduate and postgraduate trainees in order to help them develop resilience, flexibility, healthy professional identities, and a supportive medical culture.

I found this to be an excellent book, well referenced and worthy as a key resource in undergraduate and postgraduate training. It also provides important reading to remind us all what the real role of a good doctor is. We live in a world where medicine is becoming increasingly industrialised and somehow we must get back to the centre of what doctoring truly is - being with our patients.

\section{Anne Stephenson,}

GP and Director of Community Education, King's College London School of Medicine, London.

\section{E-mail: anne.stephensonakcl.ac.uk}

DOI: 10.3399/bjgp14X677626

$$
* * * * *
$$

\section{GREAT SAVES, GREAT FAILURES}

The Checklist Manifesto:

How To Get Things Right

Atul Gawande

Profile Books, 2011

PB, 224pp, £8.99, 978-1846683145

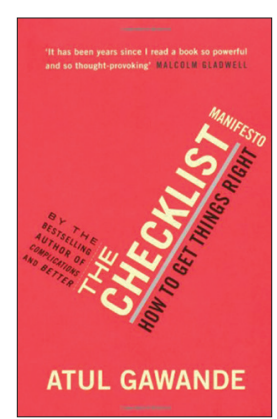

This book is for anyone who believes in the importance of checklists for the smooth running of organisations. The author, a surgeon himself, opens by discussing the banter that goes on between surgeons on their 'great saves' and 'great failures'. He ponders on why we fail in the medical world; we simply lack full understanding or either fail to apply our knowledge. Knowledge and technical sophistication have increased but what of our ability to deliver? This is where the 'checklist' comes in. According to Gawande, "expertise is the mantra of modern medicine' in this 'era of the super specialist', but despite the expertise, mistakes can still occur. Checklists first appeared in the field of aeronautics in the form of pilot's checklists. And so the author's journey of discovery begins.

After visiting people in the skyscraper industry and the restaurant and investment worlds we find Gawande working with the World Health Organization's global programme to reduce avoidable death and harm from surgery'. Alarmingly, he points out that worldwide 7 million people are left disabled and 1 million dead from surgical complications. He highlights that surgery has four big killers: infection, bleeding, unsafe anaesthesia and the unexpected. The WHO safe surgery checklist was formulated, with nineteen checks in all. Now it was time to put it to the test. It would be assessed in eight hospitals worldwide. In the Spring of 2008, the 2-minute, 19-step checklist, was implemented in the hospitals. Out of the 4000 patients studied, the checklist spared 150 people from harm and 27 from death. The findings were published in the New England Journal of Medicine in 2009.

Gawande concludes that the checklist alone isn't enough to improve safe practice but that teamwork and discipline are also crucial. Checklists also have a role beyond the operating room. Gawande's brilliance as a doctor and academic is without question, but what is really admirable is his humility, in that he has an ability to share his experiences honestly and openly with those around him, his patients, and his readers. His downto-earth style is obvious from the way he reaches out to experts in other fields to learn the 'tricks of their trade' and applies them in the medical world.

\section{Patricia McWalter,}

Consultant Family Physician. Department of Family Medicine and Polyclinics, King Faisal Specialist Hospital and Research Centre, Riyadh, Saudi Arabia.

\section{E-mail: pmcwalter/adoctors.org.uk}

\section{DOI: 10.3399/bjgp14X677635}

